\title{
Nano-scale Structure and Composition of Mixed Micelles Revealed by Small- Angle Neutron Scattering (SANS) and Molecular Dynamics (MD)
}

\author{
P. Alexandridis ${ }^{1}$, M. Tsianou², S. Kancharla ${ }^{3}$, D. Bedrov ${ }^{4}$, D. Dong ${ }^{5}$
}

${ }^{1}$ University at Buffalo, The State University of New York (SUNY) ${ }^{2}$ University at Buffalo, The State University of New York (SUNY), ${ }^{3}$ University at Buffalo, The State University of New York (SUNY), ${ }^{4}$ University of Utah, ${ }^{5}$ University of Utah

palexand@buffalo.edu

Surfactants are mixed with various ingredients during their formulation into products and during their use. The interactions of surfactants with compounds such as electrolytes, polar organic solvents and solutes, other surfactants, polymers, and, of course, solvents, especially water, are founded on thermodynamics that reflect intermolecular forces, are expressed in terms of nano-scale organization via self-assembly, and are manifested in properties such as solubilization. The macroscopic performance of surfactant formulations originates from the nanostructure attained by the surfactants in the presence of solvents and additives. Thus it is important to have information available on the structure and composition of mixed micelles at various conditions. To this end, we utilize high-resolution complementary experiments (small-angle neutron scattering, SANS, with contrast variation) and modeling (molecular dynamics, MD), and we present here examples on how the structure of micelles formed in water by anionic surfactants (sodium dodecyl sulfate, SDS, perfluorooctanoate, PFOA) responds to the presence of additives (ethanol [10.1039/d1 cp00049g], urea [10.1021/acs.langmuir.1c00433], polyethylene glycol-based polymers [10.1021/jp5023168] [10.3390/polym12081831]) across a wide range of compositions. A detailed description emerges on how the additives distribute at the outer surface of the micelles and in their interior, which is used to rationalize various properties of the mixtures.

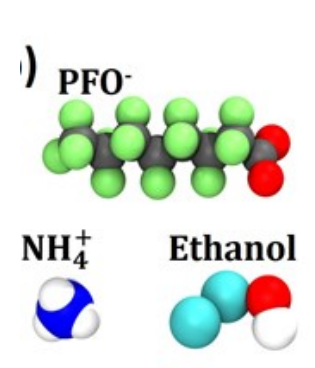

$$
\text { wt\%: } 5 \%
$$
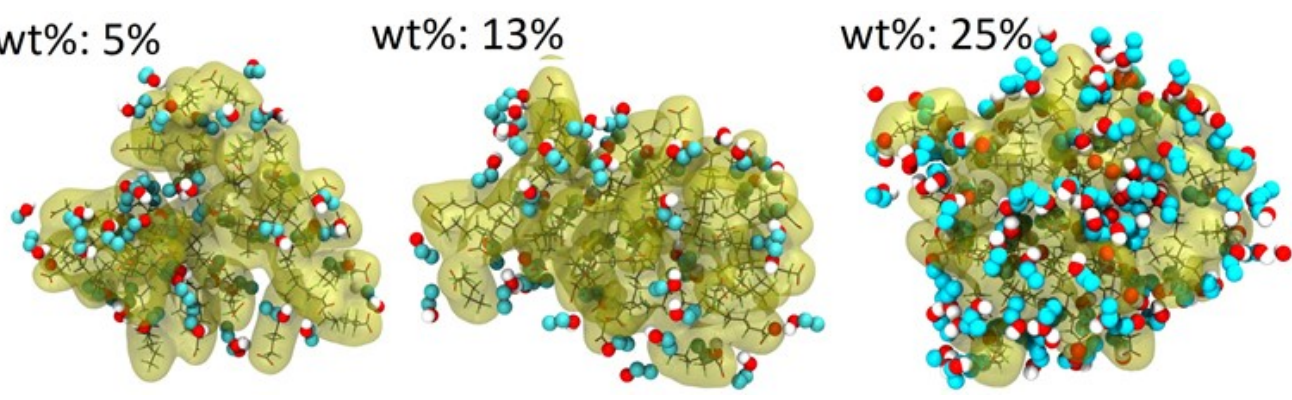

Figure 1 